\title{
MODELING OF VISCOELASTIC RESPONSE OF UNIDIRECTIONAL FIBROUS COMPOSITES MADE OF BASALT AND CARBON FIBERS
}

\author{
MICHAL ŠEJNOHA ${ }^{1}$, SOŇA VALENTOVÁ ${ }^{1}$, JAN VOREL $^{1}$, PAVEL PADEVĚT $^{1}$, RADEK SEDLÁČEK $^{2}$, \\ BLANKA TOMKOVÁ ${ }^{3} \&$ JANA NOVOTNÁ ${ }^{3}$ \\ ${ }^{1}$ Department of Mechanics, CTU in Prague, Czech Republic \\ ${ }^{2}$ Department of Biomechanics, CTU in Prague, Czech Republic \\ ${ }^{3}$ Department of Material Engineering, TU of Liberec, Czech Republic
}

\begin{abstract}
The paper is concerned with the prediction of macroscopic nonlinear viscoelastic response of unidirectional fibrous composites made of basalt and carbon fibers embedded into a polymer matrix. The objective is to derive macroscopic stress-strain curves as a function of loading rate through finite element simulations assuming a simple hexagonal arrangement of fibers in the yarn cross-section. These curves should serve as a benchmark when addressing this issue with much efficient Mori-Tanaka computational scheme, which in turn opens the way to an efficient fully coupled analysis of the complex textile geometries at the level of plies, where the Mori-Tanaka method will serve as a local stress updater at the level of individual yarns. This initial step is supported by an extensive experimental program to acquire the material parameters of the generalized nonlinear viscoelastic Leonov model describing the behavior of the polymer matrix.
\end{abstract}

Keywords: textile composites, viscoelasticity, homogenization, finite element method.

\section{INTRODUCTION}

Plain weave textile composites made of graphite fiber tows embedded in a light polymer matrix have been at the forefront of engineering interest already for some decades. Relatively recently, new types of composites exploiting relatively cheep basalt fibers have emerged as a potential substitute for more expensive carbon fibers based material systems. Limiting attention to elasticity while concentrating on various geometrical imperfections, the two systems were examined in [1]. However, moving beyond elasticity requires addressing the time dependent response of viscoelastic matrices [2]-[4].

This issue has received much attention particularly in view of various computational approaches. In this regard, two groups can essentially be identified: (1) detailed numerical simulations attempting to account for all microstructural details hidden in the formulation of various statistically equivalent periodic cells (SEPUC) [5] and (2) application of micromechanical models, e.g. the Mori-Tanaka (MT) method [6], typically enhanced to overcome to stiff predictions of macroscopic response when exceeding the limits of linear elasticity [3], [7]-[9].

In most cases, however, attention has been limited to unidirectional fibrous composites. On the other hand, practical applications call for more complex geometries such as textiles. Low material symmetry of such composites then makes difficult to formulate a simple constitutive model on macroscale. Instead, a fully coupled multi-scale analysis is usually adopted. Most often the $F E^{2}$ computational scheme [10] is used. This approach, combining detailed finite element (FEM) simulations at least at two levels, is known to be computationally very expensive. In this regard, a suitable method of attack is seen in replacing detailed finite element simulations at the level of yarns by an extremely efficient Mori-Tanaka micromechanical model. However, the applicability of the Mori-Tanaka method calls for testing to ensure that it delivers results comparable to FEM predictions. 
This paper serves as a first step in this computational endeavor by offering the macroscopic stress-strain curves provided by FEM to be compared later with the MT estimates. To this end, a simple computational model represented by a hexagonal arrangement of fibers in the yarn cross-section is sufficient. On the contrary, much attention deserves formulation and calibration of a suitable computational model pertinent to particular material systems under consideration. In this paper, two types of reinforcements, namely carbon and basalt fibers, are examined. While these are assumed linearly elastic, the matrix is expected to undergo time and rate dependent behavior well represented by the generalized Leonov model [11]. A brief introduction to theoretical bases is given in Section 2. Further details are available in [3], [9], [12]. An extensive experimental program, the essential part of this contribution, is described in Section 3. Strain based formulation of the homogenization problem based on FEM together with the results of numerical simulations is presented next in Section 4. For further details, the interested reader is referred to [4], [13]. The achieved results and potential future steps are finally summarized in Section 5.

\section{GENERALIZED LEONOV MODEL}

We begin with the Eyring flow model for the plastic component of the shear strain rate written as

$$
\frac{\mathrm{d} e_{p}}{\mathrm{~d} t}=\frac{1}{2 A} \sinh \frac{\tau}{\tau_{0}}
$$

where $A$ and $\tau_{0}$ are the material parameters to be obtained experimentally. When combined with the elastic component of the shear strain rate $\mathrm{d} e_{e} / \mathrm{d} t$ it yields the one-dimensional Leonov constitutive model [11]

$$
\frac{\mathrm{d} e}{\mathrm{~d} t}=\frac{\mathrm{d} e_{e}}{\mathrm{~d} t}+\frac{\mathrm{d} e_{p}}{\mathrm{~d} t}=\frac{\mathrm{d} e_{e}}{\mathrm{~d} t}+\frac{\tau}{\eta\left(\mathrm{d} e_{p} / \mathrm{d} t\right)},
$$

where the shear-dependent viscosity $\eta$ is provided in terms of zero shear viscosity $\eta_{0}$ (viscosity corresponding to a viscoelastic response) and stress shift function $a_{\sigma}$ as

$$
\eta\left(\mathrm{d} e_{p} / \mathrm{d} t\right)=\frac{\eta_{0} \tau}{\tau_{0} \sinh \left(\tau / \tau_{0}\right)}=\eta_{0} a_{\sigma}(\tau)
$$

where $\tau$ is the shear stress. Note that eqn (2) represents a simple Maxwell unit. An extension to a multi-dimensional behavior is simple and grounds on the generalized compressible Leonov model, equivalent to the generalized Maxwell chain model [12]. The viscosity term corresponding to the $\mu$-th unit then reads

$$
\eta_{\mu}=\eta_{0, \mu} a_{\sigma}\left(\tau_{\mathrm{eq}}\right)
$$

The equivalent shear stress $\tau_{\text {eq }}$ is provided by

$$
\tau_{\mathrm{eq}}=\sqrt{\frac{1}{2} s_{i j} s_{i j}}
$$

where $s_{i j}$ stands for the component of the deviatoric stress tensor. This also suggests a linearly elastic volumetric response thus linking the model with von Mises plasticity. The final list of constitutive equations within the limit of small strains reads 


$$
\begin{aligned}
\sigma_{m} & =K \varepsilon_{v} \\
\frac{\mathrm{d} \boldsymbol{s}}{\mathrm{d} t} & =\sum_{\mu=1}^{M} 2 \mathbf{Q} G_{\mu}\left(\frac{\mathrm{d} \boldsymbol{e}}{\mathrm{d} t}-\frac{\mathrm{d} \boldsymbol{e}_{p, \mu}}{\mathrm{d} t}\right), \quad \boldsymbol{s}=\sum_{\mu=1}^{M} \boldsymbol{s}_{\mu} \\
\boldsymbol{s}_{\mu} & =2 \mathbf{Q} \eta_{\mu} \frac{\mathrm{d} \boldsymbol{e}_{p, \mu}}{\mathrm{d} t}=2 \mathbf{Q} \eta_{0, \mu} a_{\sigma}\left(\tau_{\mathrm{eq}}\right) \frac{\mathrm{d} \boldsymbol{e}_{p, \mu}}{\mathrm{d} t}
\end{aligned}
$$

where $\sigma_{m}$ is the mean stress, $\varepsilon_{v}$ is the volumetric strain, $K$ is the bulk modulus, $G_{\mu}$ is the shear modulus of the $\mu$-th unit, and $s, e$ are the deviatoric stress and strain vectors. For general six-dimensional stress space the operator matrix $\mathbf{Q}$ is given by

$$
\mathbf{Q}=\operatorname{diag}\left[1,1,1, \frac{1}{2}, \frac{1}{2}, \frac{1}{2}\right] .
$$

Although a fully implicit integration scheme was proposed in [3] to integrate the system of eqns (6)-(8), we proceed in the footsteps of [9] and exploit a fully explicit scheme with sufficiently small time step to avoid numerical instabilities. Details on numerical implementation can be found, e.g. in [13].

\section{EXPERIMENT AND MODEL CALIBRATION}

The presented experimental program was designed to provide data for the derivation of material parameters $A, \tau_{0}$ of the Eyring flow model (1), Section 3.1, and parameters of the Maxwell chain units, the relaxation time $\theta_{\mu}$ and the spring shear modulus $G_{\mu}$ linked to zero shear viscosity $\eta_{0, \mu}=\theta_{\mu} G_{\mu}$, Section 3.2. To validate the calibrated model we compared in Section 3.3 some of the laboratory tests with numerical simulations.

\subsection{Strain rate dependent tensile tests}

For large values of parameter $A$ the Eyring flow eqn (1) can be adjusted for the case of uniaxial tension as [12]

$$
\sigma_{y}=\tau_{0} \sqrt{3} \ln (2 A \sqrt{3})+\tau_{0} \sqrt{3} \ln \dot{\varepsilon}
$$

where $\sigma_{y}$ is the yield stress, typically associated with the maximum tensile stress attained for the applied uniaxial strain rate $\dot{\varepsilon}$.

To that end, six dog bone type specimens similar to those in Fig. 1(b) and made of 285/500 "aero" Havel epoxy resin were loaded in simple tension in the displacement controlled regime at a specific strain rate until failure using the MTS Alliance $30 \mathrm{kN}$ electromechanical testing machine equipment with $30 \mathrm{kN}$ load cell. The resulting stress-strain diagrams appear in Fig. 2(a) confirming the rate dependent response of the epoxy matrix. The corresponding maximum values of the yield stress $\sigma_{y}$ are also displayed.

The evolution of strain $\varepsilon$ was measured using a clip on extensometer with an initial gauge length of $25 \mathrm{~mm}$ tightly mounted on the surface of the tested specimen, Fig. 1(a) The corresponding stresses were derived by dividing the chamber force by the average cross-section area obtained from several measurements along the specimen length, see also Section 3.2.

The Eyring eqn (10) is plotted in Fig. 2(b) as a solid line. It was obtained through a simple linear regression adopting the six experimental pairs of $\sigma_{y}-\dot{\varepsilon}$, the star points, to 


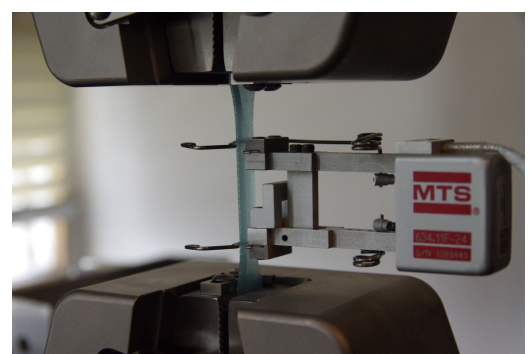

(a)

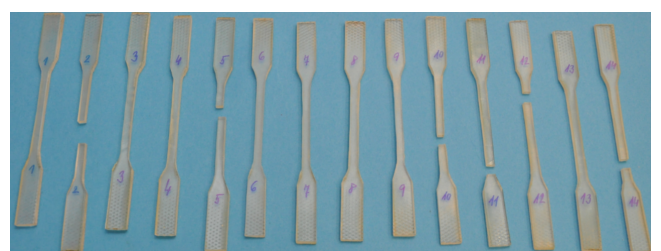

(b)

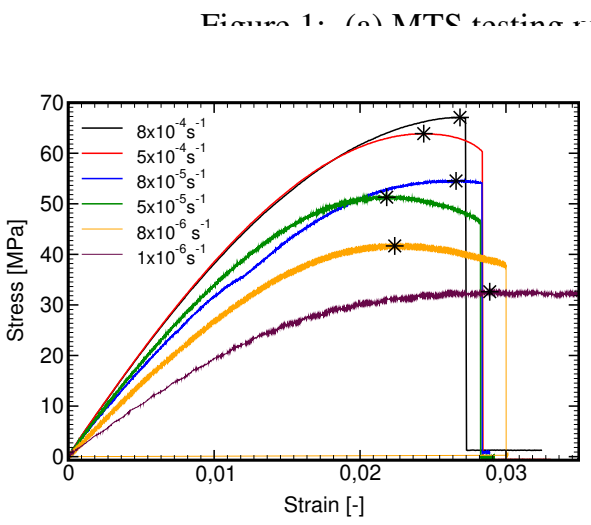

(a)

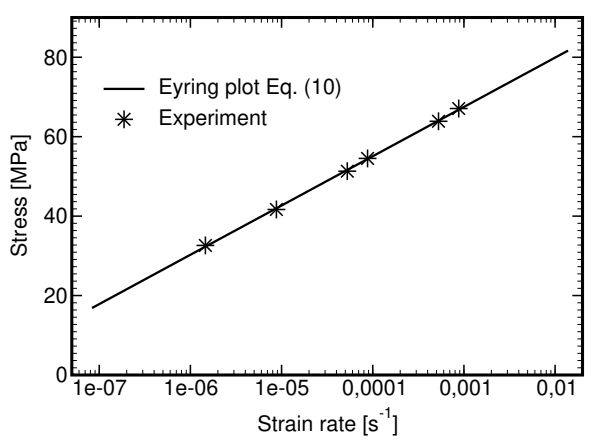

(b)

Figure 2: (a) Stress-strain curves for selected strain rates; (b) Eyring plot.

yield $A=7.928 \times 10^{7} \mathrm{~s}$ and $\tau_{0}=3.3 \mathrm{MPa}$. Point out that unlike $\tau_{0}$, which appears in the calculation of the shift factor $a_{\sigma}(\tau)$ in eqn (3), the parameter $A$ is essentially not needed in simulations.

\subsection{Stress dependent creep tests}

Standard creep tests were carried out to obtain data for the calibration of Maxwell chain model. The reason for choosing creep tests is the time-stress superposition which allows us to construct a creep compliance function for a given reference stress from a series of creep tests performed at various stress levels for a relatively short time.

The creep experiments were performed on the MTS Mini Bionix 858.02 testing system equipped with $1000 \mathrm{~N}$ load cell. In the present study, fourteen specimens plotted in Fig. 1(b) were loaded by a constant force corresponding to the required stress level evident in Fig. 3(a). The specimen preload was carried out at a constant loading speed of $500 \mathrm{Ns}^{-1}$. The strains were then recorded for two hours using again the $25 \mathrm{~mm}$ gauge length extensometer. Each test, except for tests at 40 and $45 \mathrm{MPa}$, were run twice. As seen in Fig. 3(a), the results at low stress levels (10-30MPa) are comparable thus supporting the measurement credibility. The maximum stress level was suggested based on the maximum yield stress observed in the tensile tests, recall Section 3.1. It is evident that particularly at high stress levels the acquired 

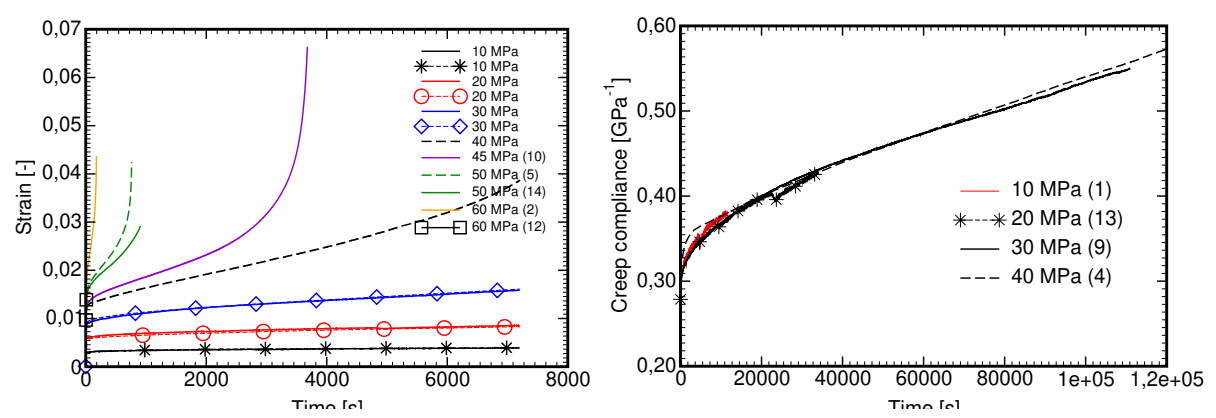

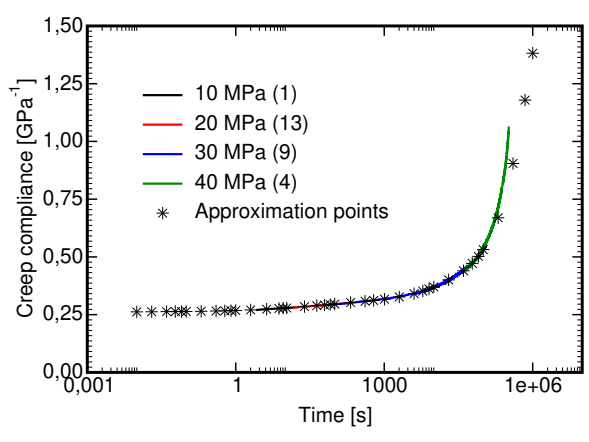

(c)

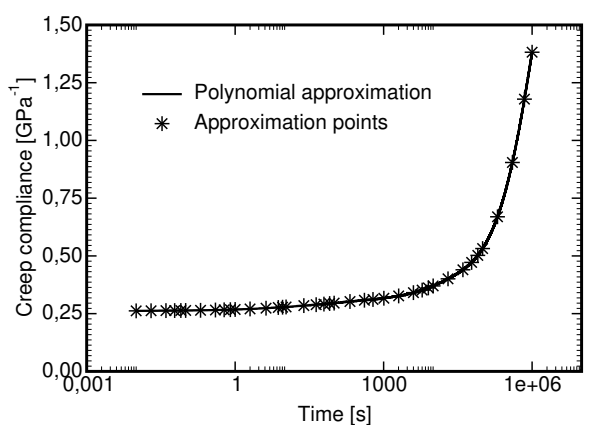

(d)

Figure 3: (a) Creep test; (b) Compliance function plotted as a function of adjusted time $\hat{t}$; (c) Master curve constructed from experiments; (d) Polynomial fit to selected approximation points based on experimental Master curve.

measurements were greatly affected by the quality of the specimen, compare the curves for 50 and $60 \mathrm{MPa}$. In addition, for stress levels exceeding 40MPa the tertiary creep can easily be identified. Exploiting these measurements would thus require a large strain formulation. Because of that, only the measurements up to $40 \mathrm{MPa}$ were adopted in further processing.

Fig. 3(b) plots the creep compliance function derived from measured strains for the selected set of specimens (numbers in parenthesis). To confirm the time-stress superposition each curve was plotted as a function of the adjusted time $\hat{t}=a_{\sigma}(\tau) t$ where $t$ represents the actual test duration. Clearly, even for stress level of $40 \mathrm{MPa}$ only data from a short initial stage of the test are applicable. This is also evident in Fig. 3 were the master curve was constructed by shifting the measurements pertinent to individual stress levels using the corresponding shift factor $a_{\sigma}(\tau)$. To span both longer and shorter times the curve was artificially prolonged as suggested by points denoted by stars. These were then used to get a smooth polynomial fit displayed in Fig. 3(d).

This smoothed approximation was employed to calibrate the Dirichlet series expansion written as

$$
J(t)=\sum_{\mu-1}^{N} J_{\mu}\left[1-\exp \left(-\frac{t}{\tau_{\mu} a_{\sigma}(t)}\right)\right], \quad R(t)=\sum_{\mu-1}^{N} E_{\mu} \exp \left(-\frac{t}{\theta_{\mu} a_{\sigma}(t)}\right)
$$


where $J(t)$ and $R(t)$ represent the creep compliance and relaxation function, respectively. First, compliances $J_{\mu}$ were determined by minimizing the least square difference of $J(t)$ given by eqn $(11)_{1}$ and experimentally derived master curve for the selected set of retardation times $\tau_{\mu}$, see Table 1 . The stiffnesses $E_{\mu}$ and relaxation times $\theta_{\mu}$ were obtained subsequently from $\left(J_{\mu}, \tau_{\mu}\right)$ pairs with the help of Laplace transform, see e.g. [13, Appendix C]. The coefficients $G_{\mu}$ needed in eqn (7) then follow directly from $E_{\mu}$ adopting constant Poisson ratio $\nu=0.39$. The results are listed in Table 1 .

Table 1: Parameters of Maxwell chain model.

\begin{tabular}{ccccc}
\hline$\mu$ & $\tau_{\mu}[\mathrm{s}]$ & $J_{\mu}\left[\mathrm{MPa}^{-1}\right]$ & $\theta_{\mu}[\mathrm{s}]$ & $E_{\mu}[\mathrm{MPa}]$ \\
\hline 1 & 0.001 & $2.606512 \mathrm{e}-04$ & $9.927397 \mathrm{e}-03$ & $2.787166 \mathrm{e}+01$ \\
2 & 0.01 & $1.905071 \mathrm{e}-06$ & $9.966502 \mathrm{e}-02$ & $1.278184 \mathrm{e}+01$ \\
3 & 0.1 & $8.808431 \mathrm{e}-07$ & $9.815126 \mathrm{e}-01$ & $7.056602 \mathrm{e}+01$ \\
4 & 1 & $4.934025 \mathrm{e}-06$ & $9.543319 \mathrm{e}+00$ & $1.711529 \mathrm{e}+02$ \\
5 & 10 & $1.276165 \mathrm{e}-05$ & $9.344254 \mathrm{e}+01$ & $2.334448 \mathrm{e}+02$ \\
6 & 100 & $1.969419 \mathrm{e}-05$ & $9.580883 \mathrm{e}+02$ & $1.418353 \mathrm{e}+02$ \\
7 & 1000 & $1.290521 \mathrm{e}-05$ & $8.275395 \mathrm{e}+03$ & $5.659977 \mathrm{e}+02$ \\
8 & 10000 & $6.291266 \mathrm{e}-05$ & $9.647045 \mathrm{e}+04$ & $1.586346 \mathrm{e}+02$ \\
9 & 100000 & $7.887707 \mathrm{e}-06$ & $2.005373 \mathrm{e}+05$ & $1.944645 \mathrm{e}+03$ \\
10 & 1000000 & $1.577867 \mathrm{e}-03$ & $4.168654 \mathrm{e}+05$ & $5.096147 \mathrm{e}+02$ \\
\hline
\end{tabular}

\subsection{Model validation through virtual laboratory tests}

To validate the Maxwell chain model, eqn $(11)_{2}$, we compared numerical simulations with experimental results of uniaxial tension discussed in Section 3.1. These comparisons for the two selected strain rates $8 \times 10^{-4}$ and $8 \times 10^{-5} \mathrm{~s}^{-1}$ appear in Fig. 4. For both loading scenarios the fit is reasonable thus supporting the Maxwell chain model to represent the time dependent resnonse of the nresent ennxv matrix

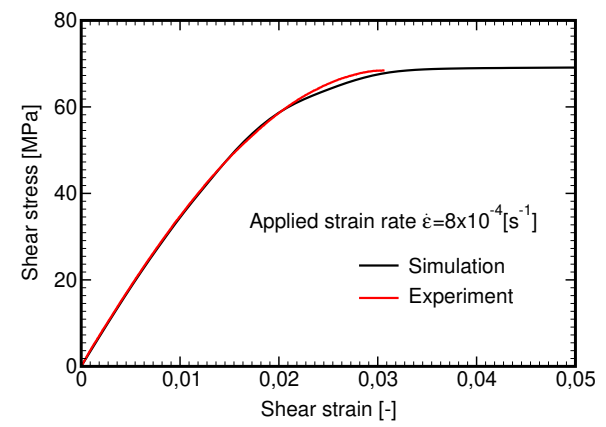

(a)

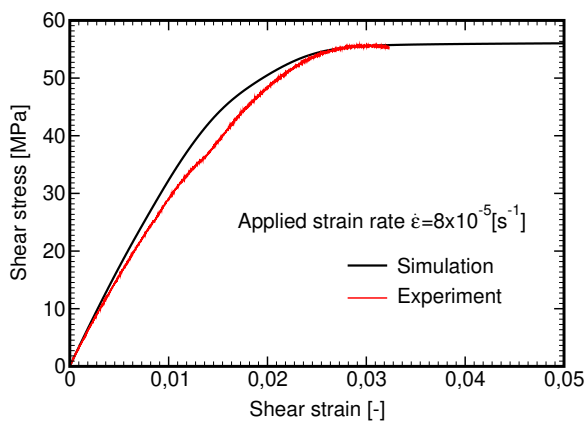

(b)

Figure 4: Comparing experiment and simulation. (a) Strain rate $\dot{\varepsilon}=8 \times 10^{-4} \mathrm{~s}^{-1}$; (b) Strain rate $\dot{\varepsilon}=8 \times 10^{-5} \mathrm{~s}^{-1}$. 


\section{HOMOGENIZATION}

It has been suggested that fibrous composites with a sufficiently large volume fraction of fibers can be assumed ergodic, statistically uniform, and thus well represented by an hexagonal arrangement of circular reinforcements in the yarn cross-section, see Fig. 5. In case of elasticity this results in a transversely isotropic material. When exceeding this limit, the SEPUC geometry yields in general an orthotropic response [4]. This is already one particular issue which cannot be captured with standard formulation of the Mori-Tanaka method.

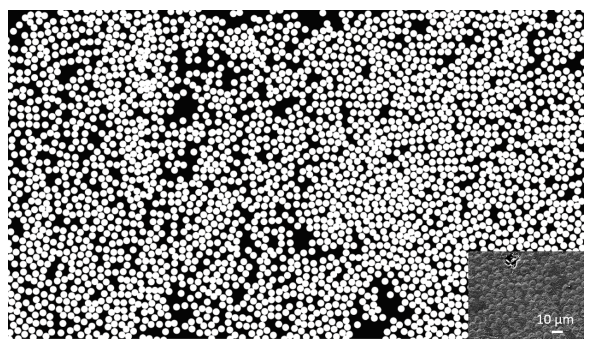

(a)

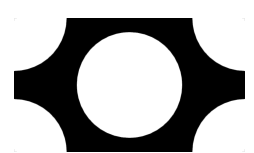

(b)

(c)

Figure 5: (a) Image of local microstructure of epoxy matrix based carbon fiber reinforced composite; (b) Periodic hexagonal array (PHA) computational model; (c) Periodic finite element mesh.

\subsection{Theoretical background}

For simplicity, we limit our attention to plane-strain formulation of the first-order homogenization problem and write the increments of local displacement and strain fields as

$$
\Delta \boldsymbol{u}(\mathrm{x})=\Delta \boldsymbol{E} \cdot \mathrm{x}+\Delta \boldsymbol{u}^{*}(\mathrm{x}), \quad \Delta \boldsymbol{\epsilon}(\mathrm{x})=\Delta \boldsymbol{E}+\Delta \boldsymbol{\epsilon}^{*}(\mathrm{x}) .
$$

Further derivation grounds on the Hill lemma, which for the displacement controlled loading conditions (the increment of macroscopically uniform strain $\Delta \boldsymbol{E}$ is prescribed) becomes

$$
\left\langle\delta \boldsymbol{\epsilon}^{\mathrm{T}} \Delta \boldsymbol{\sigma}\right\rangle=0
$$

where $\langle\cdot\rangle$ stands for volume averaging. The solution to eqn (13) is then searched in terms of the fluctuation part $\boldsymbol{u}^{*}$ of local displacements $\boldsymbol{u}$. The typical assumption of periodicity of $\boldsymbol{u}^{*}$, the same fluctuation displacements on the opposite sides of SEPUC in Fig. 5(b) and (c), then ensures that

$$
\langle\varepsilon(\mathbf{x})\rangle=\boldsymbol{E}, \quad \text { because }\left\langle\varepsilon^{*}(\mathbf{x})\right\rangle=\frac{1}{2} \int_{\Gamma}\left(n_{i} u_{j}^{*}+n_{j} u_{i}^{*}\right) \mathrm{d} \Gamma=0,
$$

where $\Gamma$ is the boundary of SEPUC and $\boldsymbol{n}$ stores the components of the unit outward normal. Adopting standard finite element discretization in eqn (13) together with local constitutive law

$$
\Delta \boldsymbol{\sigma}(\mathbf{x})=\mathbf{L}(\mathbf{x})(\Delta \varepsilon(\mathbf{x})-\Delta \boldsymbol{\mu}(\mathbf{x})), \quad \Delta \varepsilon(\mathbf{x})=\mathbf{B}(\mathbf{x}) \Delta r
$$

yields upon localization the linearized system of algebraic equations in the form

$$
\mathrm{K} \Delta r=\Delta F+\Delta f
$$


where

$$
\begin{aligned}
\mathbf{K} & =\frac{1}{\Omega} \int_{\Omega} \mathbf{B}^{\mathrm{T}}(\mathbf{x}) \mathbf{L}(\mathbf{x}) \mathbf{B}(\mathbf{x}) \mathrm{d} \Omega \\
\Delta \boldsymbol{F} & =-\frac{1}{\Omega} \int_{\Omega} \mathbf{B}^{\mathrm{T}}(\mathbf{x}) \mathbf{L}(\mathbf{x}) \boldsymbol{E} \mathrm{d} \Omega, \quad \Delta \boldsymbol{f}=\frac{1}{\Omega} \int_{\Omega} \mathbf{B}^{\mathrm{T}}(\mathbf{x}) \mathbf{L}(\mathbf{x}) \Delta \boldsymbol{\mu}(\mathbf{x}) \mathrm{d} \Omega,
\end{aligned}
$$

where $\mathbf{L}$ is the instantaneous local stiffness matrix (the stiffness matrix associated either with the fiber or the matrix phase), $\boldsymbol{\mu}$ is the eigenstrain vector here representing the creep strain developed in the polymer matrix, B is the standard geometrical matrix, and $\Delta r$ stores the increments of nodal displacements of $\boldsymbol{u}^{*}$.

Finally, the macroscopic stress increment $\Delta \boldsymbol{\Sigma}$ follows from volume averaging of local stresses $\Delta \boldsymbol{\sigma}(\mathbf{x})$, i.e.

$$
\Delta \Sigma=\langle\Delta \sigma(\mathbf{x})\rangle
$$

\subsection{Finite element simulations}

Two material systems are examined in the present study to address the influence of material properties of individual phases. The same epoxy resin was considered for both carbon and basalt fiber composite. The elastic properties of both types of fiber reinforcements were taken from $[1,14]$ and are listed in Table 2 . Since not essential for the purpose of our present study, we adopted the same volume fraction for both types of reinforcements extracted from the image of carbon fiber tow in Fig. 5(a). As for the matrix phase we recall parameters of the Maxwell chain model in Table 1.

Table 2: Material properties of individual phases.

\begin{tabular}{lcccccc}
\hline & $E_{A}$ & $E_{T}$ & $G_{A}$ & $G_{T}$ & $\nu_{A}$ & $c_{f}$ \\
& {$[\mathrm{GPa}]$} & {$[\mathrm{GPa}]$} & {$[\mathrm{GPa}]$} & {$[\mathrm{GPa}]$} & {$[-]$} & {$[-]$} \\
\hline Carbon fiber & 294 & 13 & 12 & 5 & 0.24 & 0.5 \\
Basalt fiber & 69.68 & 64.82 & 28.10 & 26.14 & 0.40 & 0.5 \\
\hline
\end{tabular}

The clear influence of fiber stiffness on the macroscopic response is evident in Fig. 6. The curves in Fig. 6(a) were constructed for pure in-plane shear loading represented by the prescribed shear strain rate $\dot{E}_{x y}=5 \times 10^{-5} \mathrm{~s}^{-1}$. The results in Fig. $6(\mathrm{~b})$ correspond to a virtual relaxation test in shear where the macroscopic shear strain $E_{x y}=0.005$ [-] was applied almost instantaneously and then held constant for 100s. Apart from macroscopic curves labeled as "composite" we also show the evolution of stress volume averages in individual phases. The observed differences are merely attributed to the differences in the transverse shear modulus where the $G_{T}$ of the basalt fiber is more than 5 times bigger than the one of the carbon fiber.

To test the influence of the geometry of SEPUC we loaded the composite in transverse direction by applying in turn the macroscopic strains $E_{x x}$ and $E_{y y}$. In particular, the cell was first preloaded with a strain rate $\dot{E}=10^{-4} \mathrm{~s}^{-1}$ up to the maximum value of $E=0.01$ [-], which was then held constant for 100s. The results for both types of material systems appear in Fig. 7. It is seen that in this particular case the creep effect is negligible, which in turn shows a negligible influence of the hexagonal arrangement of fibers. A minor difference appears for the basalt fiber based composite only, where the effect of creep is slightly more pronounced when compared to the carbon fiber based composite. Consequently, the issue 


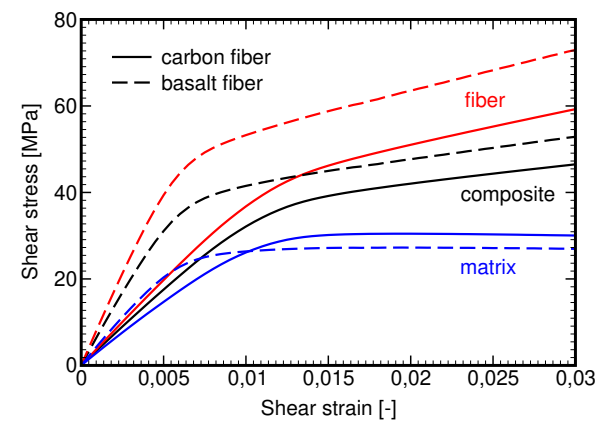

(a)

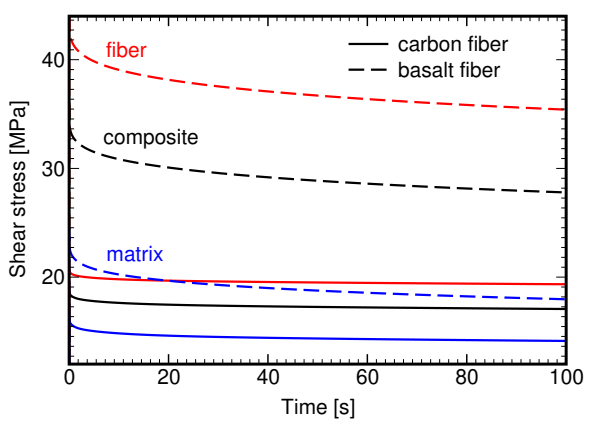

(b)

Figure 6: (a) Applied shear strain rate $\dot{E}_{x y}=5 \times 10^{-5} \mathrm{~s}^{-1}$; (b) Shear relaxation test at $E_{x y}=0.005^{\mathrm{r}} \mathrm{r}$

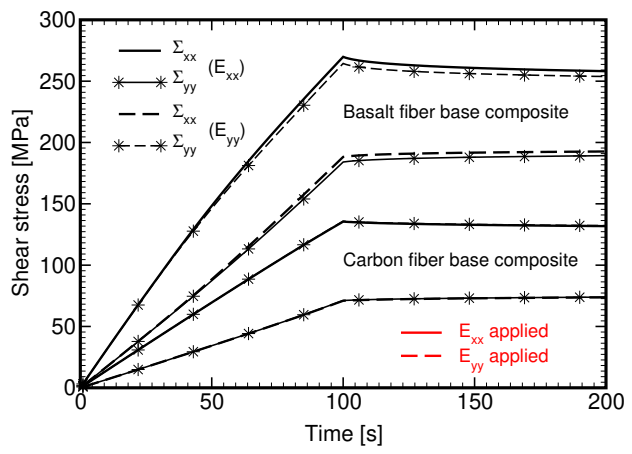

Figure 7: Modeling of transverse tension - macroscopic transverse strains $E_{x x}$ (solid lines) and $E_{y y}$ (dashed lines) applied.

of microstructure does not provide any obstacle in substituting the FEM simulation with the Mori-Tanaka method. Recall that the Mori-Tanaka method would predict, in the case of transversely isotropic fibers, a transversely isotropic response on macroscale.

\section{SUMMARY AND CONCLUSIONS}

The paper outlined an application of the generalized compressible Leonov model to predict the macroscopic response of basalt fiber and carbon fiber made composites.

Attention was accorded mainly to the calibration of the Leonov model describing the behavior of the polymer matrix made of 285/500 "aero" Havel epoxy resin. Apart from that, the selected experimental program supported the choice of this particular constitutive model. The second issue addressed in this paper was the construction of macroscopic stress-strain curves for various loading conditions.

Because these curves should provide benchmark for the application of the Mori-Tanaka micromechanical model, we adopted the first-order homogenization theory, as a reliable tool, combined with FEM simulations performed on a certain representative volume element (RVE). Herein, a simple periodic unit cell with hexagonal arrangement of fibers was 
employed. As expected, the results show a significant rate and time dependent behavior in shear, while in transverse tension this effect is less pronounced. This is mainly attributed to the model formulation corresponding to the von Mises plasticity. Consequently, the influence of RVE geometry was almost negligible for both types of composites, which may promote the applicability of the Mori-Tanaka method.

Such step, however, still needs to be supported by sufficiently accurate macroscopic predictions comparable to those provided by FEM. This would open the way to the modeling of nonlinear viscoelastic response of complex textile composites in the framework multi-scale analysis, where the efficient Mori-Tanaka method would serve as a stress updater within the homogenized, generally anisotropic, yarn. Both issues are under current investigation.

\section{ACKNOWLEDGMENTS}

The financial support provided by the GAČR Grant No. 19-15666S and by the Czech Technical University in Prague within SGS project with the application registered under the No. SGS20/038/OHK1/1T/11 is gratefully acknowledged.

\section{REFERENCES}

[1] Vorel, J., Grippon, E. \& Šejnoha, M., Effective thermoelastic properties of polysiloxane matrix based plain weave textile composites. International Journal for Multiscale Computational Engineering, 13(3), pp. 181-200, 2015.

[2] Valenta, R. \& Šejnoha, M., Epoxy resin as a bonding agent in polymer matrix composites: material properties and numerical implementation. ICCES'04, Madeira, Portugal, Jul. 26-29, 2004.

[3] Šejnoha, M., Valenta, R. \& Zeman, J., Nonlinear viscoelastic analysis of statistically homogeneous random composites. International Journal for Multiscale Computational Engineering, 2(4), pp. 645-673, 2004.

[4] Valentová, S., Šejnoha, M. \& Vorel, J., Comparing Mori-Tanaka method and firstorder homogenization scheme in the viscoelastic modeling of unidirectional fibrous composites. Acta Polytechnica CTU Proceedings, 26, pp. 133-138, 2020.

[5] Zeman, J. \& Šejnoha, M., From random microstructures to representative volume elements. Modelling and Simulation in Materials Science and Engineering, 15(4), pp. S325-S335, 2007.

[6] Benveniste, Y., A new approach to the application of Mori-Tanaka theory in composite materials. Mechanics of Materials, 6, pp. 147-157, 1987.

[7] Dvorak, G., Bahei-El-Din, Y. \& Wafa, A., The modeling of inelastic composite materials with the transformation field analysis. Modelling and Simulation in Materials Science and Engineering, 2(3A), p. 571, 1999.

[8] Masson, R., Bornert, M., Suquet, P. \& Zaoui, A., An affine formulation for the prediction of the effective properties of nonlinear composites and polycrystals. Journal of the Mechanics and Physics of Solids, 48, pp. 1203-1227, 2000.

[9] Valenta, R., Šejnoha, M. \& Zeman, J., Macroscopic constitutive law for mastic asphalt mixtures from multiscale modeling. International Journal for Multiscale Computational Engineering, 8(1), pp. 131-149, 2010.

[10] Kouznetsova, V., Geers, M.G.D. \& Brekelmans, W.A.M., Multi-scale constitutive modelling of heterogeneous materials with a gradient-enhanced computational homogenization scheme. International Journal for Numerical Methods in Engineering, 54(8), pp. 1235-1260, 2002.

[11] Leonov, A.I., Non-equilibrium thermodynamics and rheology of viscoelastic polymer media. Rheol Acta, 15, pp. 85-98, 1976. 
[12] Tervoort, T.A., Constitutive modeling of polymer glasses: Finite, nonlinear viscoelastic behaviour of polycarbonate. PhD thesis, Eindhoven University of Technology, 1996.

[13] Šejnoha, M. \& Zeman, J., Micromechanics in Practice, WIT Press: Southampton and Boston, 2013.

[14] Valentová, S., Hrbek, V., Vorel, J. \& Šejnoha, M., Strength of composite yarn under biaxial loading. Acta Polytechnica CTU Proceedings, 15, pp. 131-136, 2018. 